\title{
Correction to: PRMT1-mediated H4R3me2a recruits SMARCA4 to promote colorectal cancer progression by enhancing EGFR signaling
}

Bing Yao ${ }^{1,2+}$, Tao Gui ${ }^{1+}$, Xiangwei Zeng ${ }^{1}$, Yexuan Deng ${ }^{1}$, Zhi Wang ${ }^{1}$, Ying Wang ${ }^{1}$, Dongjun Yang ${ }^{1}$, Qixiang Li', Peipei $\mathrm{Xu}^{1}$, Ruifeng Hu${ }^{1}$, Xinyu Li ${ }^{1}$, Bing Chen ${ }^{1}$, Jin Wang ${ }^{1}$, Ke Zen ${ }^{1}$, Haitao Li $i^{3}$, Melissa J. Davis ${ }^{4}$, Marco J. Herold ${ }^{4}$, Hua-Feng Pan ${ }^{5}$, Zhi-Wei Jiang ${ }^{5}$, David C. S. Huang ${ }^{4}$, Ming Liu ${ }^{1 *}$, Junyi Ju ${ }^{1 *}$ and Quan Zhao ${ }^{1 *}$

\section{Correction to: Genome Med 13, 58 (2021)}

\section{DOI: 10.1186/s13073-021-00871-5}

In this article [1] the incorrect figure was shown as Fig. 5 and the incorrect figure appeared in Additional File 1 as Fig. S7. The figures should have appeared as shown below. The original article has been updated.

\section{Supplementary Information}

The online version contains supplementary material available at https://doi. org/10.1186/s13073-021-00966-z.

Additional file 1. Supplementary figures and related figure legends. Fig. S1. SMARCA4 binds specifically to histone H4R3me2a mark. Fig. S2. ITC assay to identify direct interactions between SMARCA4-F4 and $\mathrm{H} 4$, H4R3me2a, or H4R3me2s peptides. Fig. S3. Interation of SMARCA4 and PRMT1. Fig. S4. Identification of transcriptional targets for PRMT1 and SMARCA4 in HCT116 cells. Fig. S5. Characterization of ATAC-seq, along with ChIP-Seq of SMARCA4, H3K4me1, H3K4me3, H3K27ac in HCT116 cells. Fig. S6. PRMT1 and SMARCA4 cooperatively activate TNS4 and EGFR transcription in SW620 and HCT116 cells. Fig. S7. SMARCA4 couples with PRMT1 to promote CRC cell proliferation in SW620 and HCT116 cells. Fig. S8. AMI-1, a PRMT1 inhibitor, blocks HCT116 cell proliferation, and inhibits TNS4 and EGFR expression. Fig. S9. Combined treatment with AMI-1 and

The original article can be found online at https://doi.org/10.1186/s13073021-00871-5.

*Correspondence: liuming_nju@163.com; jujunyi@nju.edu.cn; qzhao@nju.edu.cn

${ }^{\dagger}$ Bing Yao and Tao Gui contributed equally to this work.

'The State Key Laboratory of Pharmaceutical Biotechnology, Department of Hematology, the Affiliated Drum Tower Hospital of Nanjing University Medical School, China-Australia Institute of Translational Medicine, School of Life Sciences, Nanjing University, 163 Xianlin Avenue, Nanjing 210023, China Full list of author information is available at the end of the article
Cetuximab synergistically protects Apcmin/+ mice against DSS-induced CRC progression.

\section{Author details}

${ }^{1}$ The State Key Laboratory of Pharmaceutical Biotechnology, Department of Hematology, the Affiliated Drum Tower Hospital of Nanjing University Medical School, China-Australia Institute of Translational Medicine, School of Life Sciences, Nanjing University, 163 Xianlin Avenue, Nanjing 210023, China. ${ }^{2}$ Department of Medical Genetics, Nanjing Medical University, Nanjing, China. ${ }^{3}$ Beijing Advanced Innovation Center for Structural Biology, Beijing Frontier Research Center for Biological Structure, Tsinghua-Peking Joint Center for Life Sciences, School of Medicine, Tsinghua University, Beijing, China. ${ }^{4}$ The Walter and Eliza Hall Institute of Medical Research, Department of Medical Biology, University of Melbourne, Melbourne, VIC, Australia. ${ }^{5}$ Department of General Surgery, the Affiliated Hospital of Nanjing University of Chinese Medicine, Nanjing, China.

Published online: 04 October 2021

\section{Reference}

1. Yao B, et al. PRMT1-mediated H4R3me2a recruits SMAR CA4 to promote colorectal cancer progression by enhancing EGFR signaling. Genome Med 2021;13:58. https://doi.org/10.1186/s13073-021-00871-5.

C C The Author(s). 2021 Open Access This article is licensed under a Creative Commons Attribution 4.0 International License, which permits use, sharing, adaptation, distribution and reproduction in any medium or format, as long as you give appropriate credit to the original author(s) and the source, provide a link to the Creative Commons licence, and indicate if changes were made. The images or other third party material in this article are included in the article's Creative Commons licence, unless indicated otherwise in a credit line to the material. If material is not included in the article's Creative Commons licence and your intended use is not permitted by statutory regulation or exceeds the permitted use, you will need to obtain permission directly from the copyright holder. To view a copy of this licence, visit http://creativecommons.org/licenses/by/4.0/ The Creative Commons Public Domain Dedication waiver (http://creativecommons.org/publicdomain/zero/1.0/) applies to the data made available in this article, unless otherwise stated in a credit line to the data. 


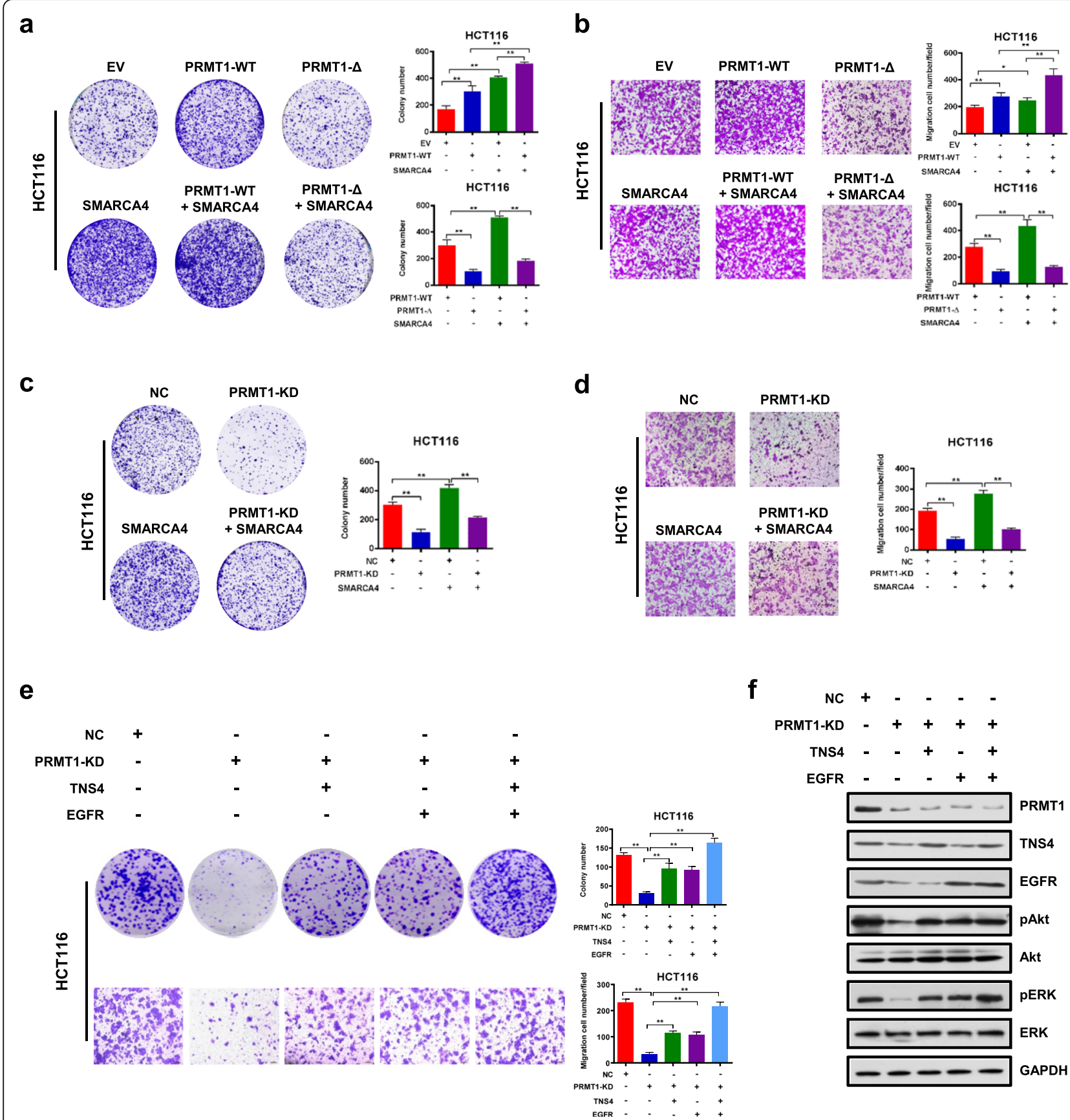

Fig. 5 SMARCA4 couples with PRMT1 to promote CRC cell proliferation through EGFR signaling in HCT116 cells. a Colony formation assay with HCT116 cells transfected with EV (empty vector, MSCV), PRMT1-WT, PRMT1- $\Delta$, SMARCA4, PRMT1-WT + SMARCA4, or PRMT1- $\Delta+$ SMARCA4. Representative images (left panels) and quantitative analyses of colony formation (right panels) are shown. $\mathbf{b}$ Cell migration assays with HCT116 cells transfected with MSCV, PRMT1-WT, PRMT1- $\Delta$, SMARCA4, PRMT1-WT + SMARCA4, or PRMT1- $\Delta+$ SMARCA4. Representative images (left panels) and quantitative analyses of the migrated cells (right panels) are shown. c Colony formation assays from NC or PRMT1-KD transfected HCT116 cells transfected or not with a SMARCA4 expression construct. Representative images (left panels) and quantitative analyses of the colony formation (right panel) are shown. $\mathbf{d}$ Cell migration assays from NC or PRMT1-KD transfected HCT116 cells transfected or not with a SMARCA4 expression construct. Representative images (left panels) and quantitative analyses of the colony formation (right panel) are shown. e Colony formation assays and cell migration assays from NC or PRMT1-KD with ectopic expression of TNS4 or EGFR, or both. Representative images (left panels) and quantitative analyses of the colony formation (right panels) are shown. $\mathbf{f}$ Western blot analysis of the expression levels of PRMT1 and EGFR signaling pathway downstream molecules p-AKT, AKT, p-ERK, and ERK in HCT116 cells with ectopic expression of TNS4 or EGFR. GAPDH served as a loading control. All results are shown as mean \pm s.d. from three independent experiments; ${ }^{*} P<0.01, * P<0.05$ compared with the indicated control 